\title{
EVALUACIÓN INSTRUMENTAL DE LA TEXTURA DEL QUESO ELABORADO CON SUERO CONCENTRADO POR ULTRAFILTRACIÓN
}

\author{
César Guerrero Ramos ${ }^{\mathrm{a}}$, Walter F. Salas Valerio ${ }^{\mathrm{a}}$ y Edwin O. Baldeón Chamorro ${ }^{\mathrm{a}^{*}}$
}

\begin{abstract}
RESUMEN
El suero de leche es un residuo líquido de la industria láctea, el cual es eliminado por los fabricantes de queso; la concentración de éste mediante ultrafiltración ha sido una alternativa para su aprovechamiento. Los objetivos del presente trabajo fueron: Obtener un queso a partir del suero de leche concentrado por ultrafiltración y evaluar instrumentalmente la textura del queso por los métodos de análisis de perfil de textura (TPA) y compresión uniaxial. En el presente trabajo se concentró el suero de leche en un sistema de ultrafiltración de capacidad de $5 \mathrm{~L}$ bajo las siguientes condiciones: presión de 2,5 bar, temperatura de $25^{\circ} \mathrm{C}$, flujo de salida del permeado de $0,33 \mathrm{~kg} / \mathrm{h}$ y membrana cerámica tubular de un tamaño de poro de 50 nm. El suero concentrado se utilizó en la elaboración del queso con diferentes porcentajes de sustitución y se hizo una evaluación instrumental de la textura; como resultado de evaluación, los quesos presentaron características de textura blanda con bajo contenido en grasa.
\end{abstract}

Palabras clave: Queso fresco, suero de leche, TPA, compresión uniaxial.

\section{INSTRUMENTAL EVALUATION A CHEESE TEXTURE MADE FROM CONCENTRATED WHEY BY ULTRAFILTRATION}

\begin{abstract}
The whey is a liquid residue of the dairy industry which is removed by cheese makers; concentration using ultrafiltration has been an alternative to its use. The objectives of this study were produce a cheese from whey concentrated by ultrafiltration and instrumentally evaluate cheese texture using the texture profile analysis (TPA) and uniaxial compression. In this paper the whey is concentrated on an ultrafiltration system capacity of $5 \mathrm{~L}$ under the following conditions: pressure of 2,5 bar, $25^{\circ} \mathrm{C}$, the permeate outflow of $0,33 \mathrm{~kg} / \mathrm{h}$ ceramic membrane with pore size of $50 \mathrm{~nm}$. The concentrated whey used in the manufacture of cheese using different percentages of replacement and they were evaluated; the results showed cheeses soft with low fat.
\end{abstract}

Key words: Cheese, whey, TPA analysis, uniaxial compression.

\section{INTRODUCCIÓN}

Últimamente se ha descubierto las bondades de las proteínas del suero y de otros componentes por su valor nutritivo y funcional. El suero de leche ha sido eliminado como un flujo de residuo por los fabricantes de queso y de caseína. Las empresas queseras han realizado la mejor gestión económica para eliminar el suero, considerado como un producto de desecho con muy poco valor. ${ }^{1}$

\footnotetext{
${ }^{a}$ Ingeniero en Industrias Alimentarias; trabaja en Ecolab Peru Holdings S.R.L.

b. Docente principal de la Facultad de Industrias Alimentarias de la Universidad Nacional Agraria La Molina. Lima, Perú.

${ }^{\text {c }}$ Docente asociado del a Facultad de Industrias Alimentarias de la Universidad Nacional Agraria La Molina. Lima. Perú. Av. La Molina S/N, Lima 12, Perú, Teléfono: +5113495764. eobch@1amolina.edu.pe
} 
A pesar del gran número de variedades de queso, la industria está continuamente buscando nuevas tecnologías e ingredientes para mejorar: la vida útil, la apariencia, la textura, la sensación en la boca y el gusto por el uso de ingredientes innovadores. Por ejemplo, la ultrafiltración (UF) se ha utilizado desde los años 70 como una tecnología clave para mejorar las características del queso ${ }^{2}$. La operación de ultrafiltración ha permitido la integración de las proteínas del suero con la caseína y los componentes de la grasa dentro de la cuajada con el fin de mejorar el valor nutricional del queso ${ }^{3}$. La ultrafiltración es una herramienta muy utilizada industrialmente para separar las proteínas del suero; es aprovechada para elaborar diversos alimentos en las industrias alimentarias. En el proceso de ultrafiltración del suero de leche se separan en dos constituyentes: El retenido (proteínas del lactosuero) y el permeado (constituido por sales, agua y la mayor parte de lactosa) ${ }^{4}$.

Durante la elaboración del queso, las proteínas séricas inicialmente son retenidas en la cuajada, pero al ser solubles, son eliminadas en gran parte en el suero durante el corte de la misma; consecuentemente sólo quedan retenidas en el queso del 3 al 5\% del total de las proteínas séricas ${ }^{5}$. Esta proporción, constituye un reservorio de aminoácidos utilizados para el desarrollo del sabor y aroma. Las proteínas séricas no dan lugar a coágulos elásticos y retráctiles, como la caseína, por lo que tienden a retener mayor proporción de agua ${ }^{6}$.

El Análisis de Perfil de Textura (TPA) es uno de los métodos instrumentales más ampliamente utilizado para la evaluación de textura del queso ${ }^{7}$. El método de análisis de perfil de textura, es un procedimiento instrumental para medir y cuantificar la dureza, la gomosidad, etc; éstos están influenciados por la velocidad de deformación del alimento cuando éste es sometido al análisis instrumental y está demostrado que correlacionan bien con los características o atributos en la evaluación sensorial. Los objetivos que se plantearon en el presente trabajo fueron: Obtener un queso a partir del suero de leche concentrado por ultrafiltración y evaluar instrumentalmente la textura del queso por los métodos de TPA y compresión uniaxial.

\section{Lugar de ejecución}

\section{PARTE EXPERIMENTAL}

El presente trabajo se realizó en los laboratorios de Fisico-Química, ingeniería de alimentos, investigación, planta piloto de alimentos y planta piloto de leche de la Facultad de Industrias Alimentarias de la Universidad Nacional Agraria La Molina (UNALM).

\section{Materiales y reactivos}

Muestra. Se utilizó leche y lactosuero procedente de la planta piloto de leche de la UNALM. El queso fresco "La Molina" se adquirió de la Planta Piloto de Leche que sirvió como referencia.

Reactivos. Ácido nítrico $\left(\mathrm{HNO}_{3}\right), 69$-70\%. BEAKER ANALYZED ACS Reagent. Hidróxido de sodio $(\mathrm{NaOH})$, Fermont, Productos químicos Monterrey S.A. de C.V.

Equipo utilizado. Ultrafiltrador DELTALAB, modelo MP351 que cuenta con filtros de 50 y $20 \mathrm{~nm}$, trabaja a una presión máxima de 3 bares. 


\section{Método de análisis}

Análisis químico proximal de la materia prima

- Grasa, por el método de Gerber ${ }^{8}$.

- Contenido de humedad, método gravimétrico ${ }^{9}$.

- Proteínas, por el método semi micro Kjendahl. Se utilizó factor de conversión de nitrógeno a proteína de $6,38^{9}$.

- Cenizas ${ }^{9}$.

- Sólidos totales, método gravimétrico ${ }^{9}$

\section{Análisis físico químico para el suero}

- $\quad$ Acidez, densidad y grasa (INDECOPI, 1998)

- Sólidos totales y $\mathrm{pH}^{10}$.

\section{Análisis del perfil de textura (TPA) del queso}

El análisis de perfil de textura (TPA) del queso ${ }^{11}$ se realizó bajo las siguientes condiciones:

- Dimensiones de la muestra: $20 \mathrm{~mm}$ de altura (L) x $20 \mathrm{~mm}$ de diámetro.

- Se utilizó un accesorio cilíndrico de prueba de $40 \mathrm{~mm}$ de diámetro.

- Compresión: $20 \%$ de deformación.

- Velocidad a $10 \mathrm{~mm} / \mathrm{min}$.

- $\quad$ Temperatura de las muestras: $4^{\circ} \mathrm{C} \pm 2,0^{\circ} \mathrm{C}$.

- Tiempo de ejecución: menor a 180 segundos.

- Se realizó 5 repeticiones.

\section{Análisis de compresión uniaxial del queso}

El análisis de compresión uniaxial ${ }^{12,13}$. Se procedió bajo las siguientes condiciones:

- Dimensiones de la muestra: $20 \mathrm{~mm}$ de altura x $20 \mathrm{~mm}$ de diámetro.

- Se utilizó un accesorio cilíndrico de prueba de $40 \mathrm{~mm}$ de diámetro.

- Compresión: $75 \%$ de deformación.

- Velocidad a $100 \mathrm{~mm} / \mathrm{min}$.

- $\quad$ Temperatura de las muestras: $4^{\circ} \mathrm{C} \pm 2,0^{\circ} \mathrm{C}$.

- Tiempo de ejecución: menor a los 15 segundos.

- Se realizó 5 repeticiones.

\section{Propiedades reológicas del queso}

El esfuerzo normal $(\sigma)$ se determinó dividiendo los resultados de la carga $(P)$ entre el área transversal de la muestra, expresada en $\mathrm{kN} / \mathrm{m}^{2}$. La deformación unitaria $(\varepsilon)$, se calculó dividiendo la deformación total $(\delta)$ entre la longitud (L) inicial de la muestra.

Con los diferentes valores de esfuerzo $(\sigma)$ y deformación unitaria $(\varepsilon)$, se construye la curva de esfuerzo y deformación.

\section{Metodología experimental}

\section{Obtención del lactosuero concentrado}

El suero de leche se filtró usando una malla de material sintético de 0,1 $\mathrm{mm}$ de diámetro para 
retener los restos de partículas de queso y grasa. Para la concentración del suero se empleó un sistema de ultrafiltración (DELTALAB, modelo MP351) de capacidad de 5 L; se mantuvo a una presión de 2,5 bar, a una temperatura de $25^{\circ} \mathrm{C}$ y a un flujo de salida del permeado de $0,33 \mathrm{~kg} / \mathrm{h}$. El suero pasa a través de una membrana cerámica tubular de un tamaño de poro de $50 \mathrm{~nm}$, saliendo dos flujos: un flujo de permeado y otro flujo de retenido; este último se recircula por medio de una bomba hasta obtener un factor de retención volumétrica (FRV) de 2; finalmente, el suero queda concentrado siendo utilizado para la elaboración del queso.

\section{Elaboración del queso fresco}

La leche se pasteurizó a $63^{\circ} \mathrm{C}$ por $30 \mathrm{~min}$; se dejó enfriar hasta $40^{\circ} \mathrm{C}$, luego se adicionó el cuajo $\left(1,5 \mathrm{~g} / 100 \mathrm{~L}\right.$ leche) para que coagule; se dejó en reposo a una temperatura $40^{\circ} \mathrm{C}$. Se procedió al corte de la cuajada usando un cortador (liras) y se dejó en reposo por espacio de 10 minutos; seguidamente se realizó el desuerado con agitación continua; el primer desuerado se realizó a 1/3 de su volumen por espacio de 15 minutos, y para completar el desuerado se adicionó agua caliente a $65^{\circ} \mathrm{C}$, elevando la temperatura de la cuajada a $38^{\circ} \mathrm{C}$. A la cuajada obtenida se mezcló con $1,25 \%$ de sal y $0,05 \%$ de sorbato de potasio. Para el moldeado del queso, la cuajada se recolectó en moldes plásticos asépticos, ejerciendo una mínima presión se escurrió una cantidad de suero impregnada en los granos de la cuajada. Luego de $24 \mathrm{~h}$ en refrigeración se retiró los moldes. Finalmente, se embolsó en bolsas de polietileno de alta densidad y se mantuvo en refrigeración a $5{ }^{\circ} \mathrm{C}$ hasta su posterior análisis de textura.

\section{Diseño experimental para la elaboración del queso con suero concentrado por ultrafiltración}

La leche fresca y el suero concentrado fueron mezcladas en proporciones de $10 \%, 20 \%, 30 \%$ y $50 \%$; la mezcla obtenida se empleó para la elaboración del queso siguiendo el procedimiento descrito anteriormente. En los quesos obtenidos se evaluó la textura por los métodos TPA y compresión uniaxial.

\section{Análisis estadístico}

En evaluación instrumental de la textura del queso obtenido con suero de leche concentrada por ultrafiltración, se analizó mediante un diseño completo al azar (DCA) al 95\% de significancia, con cinco repeticiones, teniendo como único factor la concentración y como variable de respuesta la textura. Para ver la diferencia entre los tratamientos, se realizó la prueba de comparación DLS al 95\% de significancia. Para el análisis estadístico se utilizó STATGRAPHICS plus vs. 5.1.

\section{RESULTADOS Y DISCUSIÓN \\ Composición proximal del suero de leche, retenido y permeado}

En el tabla 1 se muestra la composición del suero de leche retenido y permeado. Los porcentajes de proteína y humedad del suero utilizado como materia prima fueron cercanos a los valores reportados ${ }^{14}$, siendo para la humedad del suero valores entre 93 a $94 \%$ y para la proteína valores entre 0,8 a $1 \%$. El porcentaje de grasa del suero permeado en el estudio realizado fue de $0 \%$ que coincide con el valor reportado ${ }^{15}$. El porcentaje de proteína del suero 
permeado en el estudio fue de $0 \%$; en cambio ${ }^{15}$ reportan valores de proteína cruda de $0,25 \%$.

Tabla 1. Composición proximal del suero de leche, retenido y permeado.

\begin{tabular}{lccc}
\hline $\begin{array}{c}\text { Componentes } \\
(\%)\end{array}$ & Suero & $\begin{array}{c}\text { Suero } \\
\text { retenido }\end{array}$ & $\begin{array}{c}\text { Suero } \\
\text { permeado }\end{array}$ \\
\hline Grasa & 0,3 & 0,7 & 0 \\
Humedad & 93,32 & 91,82 & 95,09 \\
Extracto seco & 6,68 & 8,18 & 4,91 \\
Proteínas & 0,8 & 4,11 & 0 \\
\hline
\end{tabular}

Valores promedio de tres repeticiones.

\section{Análisis fisicoquímico del suero de leche, retenido y permeado.}

En la tabla 2 se muestra el análisis del suero de leche, retenido y permeado. El porcentaje de sólidos totales del suero permeado en el estudio fue de 6,39\%; cercano al valor de sólidos totales reportado que fue de $6,02 \%{ }^{15}$. El pH del suero utilizado fue de 6,68 , similar al valor de $\mathrm{pH}$ reportado que fue de $6,45^{16}$.

Tabla 2. Análisis fisicoquímico del suero de leche, retenido y permeado.

\begin{tabular}{lccc}
\hline Componentes & Suero & $\begin{array}{c}\text { Suero } \\
\text { retenido }\end{array}$ & $\begin{array}{c}\text { Suero } \\
\text { permeado }\end{array}$ \\
\hline Acidez $\left({ }^{\circ} \mathrm{D}\right)$ & 1,15 & 1,35 & 1,46 \\
$\mathrm{pH}$ & 6,68 & 6,57 & 5,63 \\
Densidad $(\mathrm{g} / \mathrm{ml})$ & 1,03 & 1,03 & 1,03 \\
Sólidos totales $(\%)$ & 7,25 & 8,61 & 6,39 \\
\hline
\end{tabular}

Valores promedio de tres repeticiones.

\section{Composición proximal y análisis fisicoquímico del queso fresco y de los tratamientos}

En la tabla 3 se aprecia la composición proximal y análisis fisicoquímico del queso fresco y de los tratamientos. Los valores de grasa $(24,08 \%)$, proteína $(18,82 \%)$ y humedad $(53,2 \%)$ para el queso fresco están dentro de los valores establecidos ${ }^{17}$. En el queso con $10 \%$ de suero concentrado presentó una diferente composición en los valores de grasa $(20,5 \%)$ y humedad $(24,8 \%)$. Por otro lado, al aumentar el porcentaje de sustitución de suero concentrado, los 
quesos mostraron diferente composición química; es el caso del queso con 50\% de suero concentrado, los valores de grasa, proteína y humedad, disminuyeron en $78 \%, 26,7 \%$ y $29 \%$, respectivamente, comparados con el queso fresco.

Tabla 3. Composición proximal y análisis fisicoquímico de las muestras tratadas.

\begin{tabular}{llllll}
\hline & \multicolumn{5}{c}{ Tratamientos } \\
\cline { 2 - 6 } & $\begin{array}{l}\text { Queso } \\
\text { fresco }\end{array}$ & $\begin{array}{l}\text { Queso } \\
\text { con 10\% }\end{array}$ & $\begin{array}{l}\text { Queso } \\
\text { con 20\% }\end{array}$ & $\begin{array}{l}\text { Queso } \\
\text { con 30\% }\end{array}$ & $\begin{array}{l}\text { Queso } \\
\text { con 50\% }\end{array}$ \\
Composición (\%) & & S.C. & S.C. & S.C. & S.C. \\
\hline Grasa & 24,08 & 20,5 & 12,5 & 8,5 & 5,3 \\
Proteínas & 18,82 & 19,14 & 16,06 & 15,91 & 13,79 \\
Humedad & 53,2 & 24,8 & 34,01 & 36,79 & 37,75 \\
Cenizas & 3,1 & 3,99 & 2,19 & 2,15 & 2,14 \\
Acidez & 0,65 & 0,45 & 0,4 & 0,24 & 0,19 \\
pH & 6,6 & 6,7 & 6,96 & 5,14 & 5,27 \\
\hline
\end{tabular}

Valores promedio de tres repeticiones. S.C. significa suero concentrado.

\section{Evaluación de la textura del queso}

Los quesos son productos poco quebradizos para los cuales el TPA ha sido ampliamente usado y donde la dureza y la elasticidad son parámetros determinantes de la evaluación de la textura ${ }^{18}$. Para la evaluación de la textura de las muestras se propuso dos métodos: TPA y compresión uniaxial.

\section{Método TPA}

En la tabla 4 se muestra los resultados de TPA para los 5 tratamientos realizados. La dureza disminuyó en $59,4 \%$ en el queso con $10 \%$ de suero concentrado y disminuyó en $87,7 \%$ en el queso con $50 \%$ de suero concentrado, comparado con el queso fresco (ver figura 1); lo contrario ocurrió con la adhesividad que se incrementó en $78,6 \%$ para el queso con $10 \%$ de suero concentrado y llegó a incrementar hasta 3,21 veces para el queso con 30\% de suero concentrado respecto al queso fresco.

Tabla 4. Caracterización de la textura de las muestras por el método de TPA.

\begin{tabular}{lllll}
\hline Tratamientos & Dureza $(\mathbf{N})$ & $\begin{array}{l}\text { Elasticidad } \\
(\mathbf{m m})\end{array}$ & Cohesividad & $\begin{array}{l}\text { Adhesividad } \\
(\mathbf{N})\end{array}$ \\
\hline Queso “La Molina" & $3,05^{\mathrm{a}} \pm 0,50$ & $3,28^{\mathrm{b}} \pm 0,11$ & $0.75^{\mathrm{ab}} \pm 0,01$ & $-0,16^{\mathrm{a}} \pm 0,10$ \\
Queso fresco & $3,25^{\mathrm{a}} \pm 0,34$ & $3,73^{\mathrm{a}} \pm 0,03$ & $0,77^{\mathrm{a}} \pm 0,03$ & $-0.14^{\mathrm{ab}} \pm 0,12$ \\
Queso con $10 \%$ S.C. & $1,32^{\mathrm{b}} \pm 0,16$ & $3,20^{\mathrm{bc}} \pm 0,14$ & $0,74^{\mathrm{abc}} \pm 0,00$ & $-0,25^{\mathrm{bc}} \pm 0,04$ \\
Queso con 20\% S.C. & $1,22^{\mathrm{b}} \pm 0,24$ & $3,44^{\mathrm{ab}} \pm 0,10$ & $0,66^{\mathrm{c}} \pm 0,00$ & $-0,34^{\mathrm{cd}} \pm 0,07$ \\
Queso con 30\% S.C. & $0,47^{\mathrm{c}} \pm 0,14$ & $2,73^{\mathrm{d}} \pm 0,33$ & $0,71^{\mathrm{bc}} \pm 0,00$ & $-0,45^{\mathrm{d}} \pm 0,10$ \\
Queso con 50\% S.C. & $0,40^{\mathrm{c}} \pm 0,16$ & $2,87^{\mathrm{cd}} \pm 0,55$ & $0,77^{\mathrm{c}} \pm 0,02$ & $-0,15^{\mathrm{ab}} \pm 0,05$ \\
\hline
\end{tabular}

Diferentes letras en una misma columna; diferencias significativas p-value $<0.05$ 


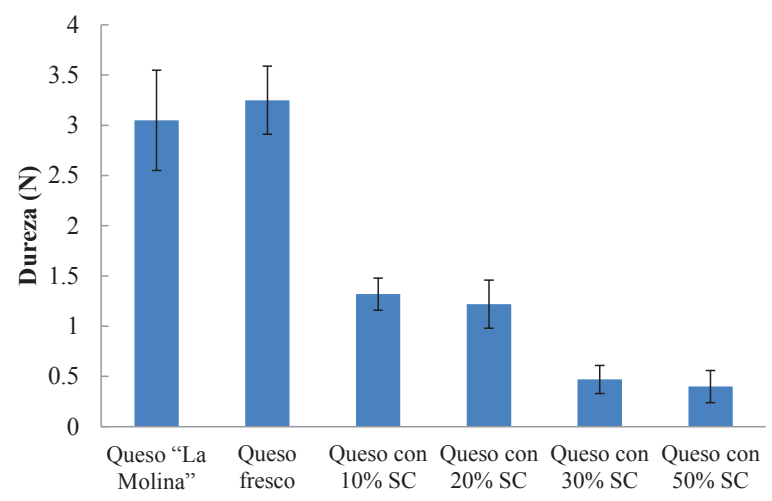

Figura 1. Parámetro de dureza determinado por el método de TPA

La dureza del queso disminuyó con el incremento del porcentaje de suero concentrado por ultrafiltración, haciendo que los quesos tengan una apariencia de textura blanda y suave, y bajos contenido de proteína y grasa (ver tabla 3). La proteína de suero desnaturalizada genera una textura menos firme que la caseína, lo que podría explicar la suave y débil textura del queso ${ }^{7}$.

\section{Método de compresión uniaxial}

En la tabla 5 se muestra los resultados de la compresión para los 5 tratamientos realizados. Existe una disminución de la dureza en $63,7 \%$ para el queso con $10 \%$ de suero concentrado y en $85,9 \%$ para el queso con $50 \%$ de suero concentrado comparado con el queso fresco. Comparando las pruebas de TPA y compresión uniaxial se encontró las siguientes diferencias: En la prueba de TPA se determinó un valor de dureza de 3,25 $\mathrm{N}$ y en la prueba de compresión uniaxial un valor de 25,9 $\mathrm{N}$ para el queso fresco; esta diferencia se debe al porcentaje de compresión aplicado a cada prueba; en la compresión uniaxial fue de $75 \%$ y en el TPA fue de $20 \%$.

Tabla 5. Determinación de la dureza por el método de compresión uniaxial.

\begin{tabular}{ll}
\hline Tratamientos & Dureza (N) \\
\hline Queso “La Molina” & $24,30^{\mathrm{a}} \pm 2,826$ \\
Queso fresco & $25,90^{\mathrm{a}} \pm 2,939$ \\
Queso con $10 \%$ S.C. & $9,40^{\mathrm{b}} \pm 1,820$ \\
Queso con $20 \%$ S.C. & $9,00^{\mathrm{b}} \pm 0,999$ \\
Queso con $30 \%$ S.C. & $8,76^{\mathrm{b}} \pm 2,258$ \\
Queso con $50 \%$ S.C. & $3,66^{\mathrm{c}} \pm 1,143$
\end{tabular}

$\overline{\text { Diferentes letras en una misma columna; diferencias }}$ significativas $p$-value $<0.05$ 
Por otro lado, el método de TPA es utilizado para determinar los parámetros de textura en los quesos; en cambio, en el método de compresión uniaxial es empleado para determinar propiedades reológicas de los quesos.

\section{Propiedades reológicas del queso}

El queso es un material altamente viscoelástico, cuya relación esfuerzo-deformación depende significativamente del factor tiempo y cuyas propiedades reológicas están determinadas por la composición química del producto ${ }^{19}$. En la figura 2 se muestra la curva típica de una prueba de esfuerzo y deformación obtenida mediante la prueba de compresión uniaxial. A medida que la deformación se incrementa el queso tienen un comportamiento viscoelástico hasta un pico máximo en el cual el queso sufre una fractura; después de este punto el esfuerzo decrece a pesar de seguir aumentando la deformación; es decir, el queso pierde su resistencia a la carga aplicada. Este comportamiento se observó en los quesos frescos y en el queso con $10 \%$ suero concentrado.

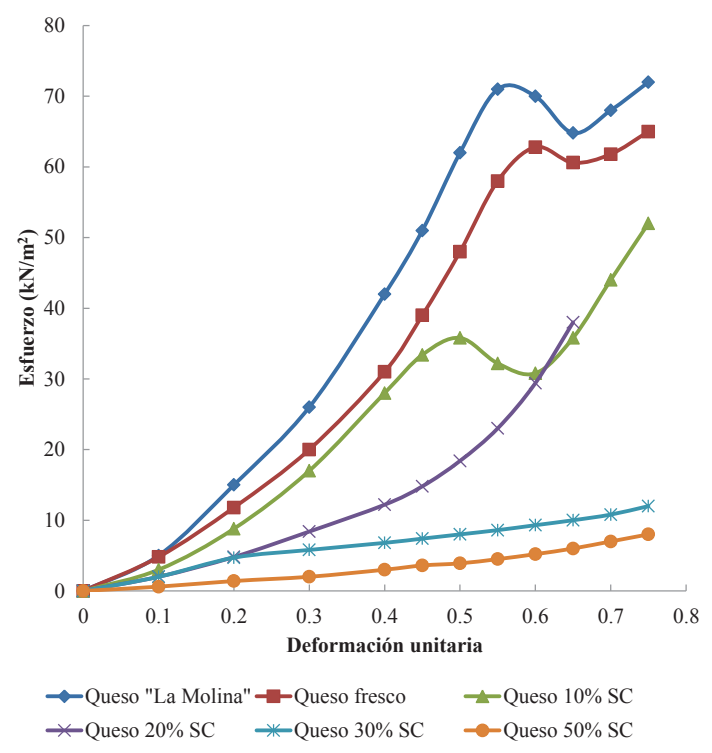

Figura 2. Curva de esfuerzo y deformación promedio de los tratamientos

Los materiales duros se caracterizan porque a una gran fuerza de cizalla produce una deformación relativamente pequeña, mientras que para materiales blandos una gran fuerza de cizalla produce una gran deformación ${ }^{20}$. El queso fresco y el queso con $10 \%$ de suero concentrado presentaron una deformación pequeña por lo que fueron considerados quesos blandos y los quesos obtenidos con $20 \%, 30 \%$ y $50 \%$ de suero concentrado tuvieron características de quesos blandos débiles, de apariencia cremosa y bajos en grasas (ver tabla 3); éstas últimas son parecida a una tipo de queso elaborado con suero de leche bajo en $\operatorname{grasa}^{21}$. 


\section{CONCLUSIONES}

Fue posible la elaboración de queso fresco utilizando suero concentrado por ultrafiltración (presión de 2,5 bar, temperatura de $25^{\circ} \mathrm{C}$, tamaño de filtro de $50 \mathrm{~nm}$ y un factor de retención volumétrica de 2).

La dureza de los quesos analizados disminuyó con el aumento de la proporción de suero concentrado por ultrafiltración, tanto en la prueba de TPA como por la compresión uniaxial. El queso elaborado con $10 \%$ de suero concentrado presentó una textura blanda y suave; en cambio, los quesos con más de $10 \%$ de suero concentrado presentaron texturas débiles con bajo contenido en grasa.

\section{AGRADECIMIENTO}

Los autores agradecen a la Planta Piloto de Leche de la Universidad Nacional Agraria La Molina por la orientación y realización de las primeras pruebas.

\section{BIBLIOGRAFÍA}

1. Smithers, G.W. Whey and whey proteins-From 'gutter-to-gold'. International Dairy Journal. 2008; 18 (7): 695-704.

2. Shell, J. Ultrafiltration in cheese making. In Proceedings of the Italian Cheese Seminar, 16-18 September, Madison, Wisconsin, USA 1986.

3. Hinrichs, J. Incorporation of whey proteins in cheese. International Dairy Journal. 2001; 11: 495-503.

4. Tetra Pak Processing Systems AB. "Manual de Industrias Lácteas". Madrid Vicente ediciones. España, 1996.

5. Johnson, M. "Cheese products. IN: Applied Dairy Microbiology". Ed. Elmer Marth y James Steele. New York. 1998, 213 - 250.

6. Scott. R. Fabricación de queso. Editorial ACRIBIA. Zaragoza, España, 1991, 520.

7. Arteaga, M. "Evolución de la maduración del queso chanco elaborado con adición de suero en polvo". Tesis para optar el grado de Magíster en Ciencias y Tecnología de la Leche. Universidad Austral de Chile, Valdivia, Chile, 2004.

8. Pinto, M., Vega y Leon, S. y Perez, N. "Métodos de análisis de la leche y derivados". Imprenta Universitaria, S.A. Valdivia, Chile, 1998, 489.

9. AOAC. Internatinonal Association of Official Aanalytical Chemists. Oficial methods of analysis. EEUU, 1995.

10. Pinto, M., Rojas, M. Israel, L. y Molina, H. Exactitud del Método de Gerber para la Determinación de Materia Grasa en Leche. Arch. Med. Vet. Universidad Austral de 
Chile, 1976; 8(2): 83 - 89.

11. Irudayaraj, J. Chen, M., and McMahon, D. J. Texture development in cedar cheese during ripening, Can. Agric. Eng, 1999; 41: 253-258.

12. Fontecha, J. Kaláb, M. Medina, J. Peláez, C. Juárez, M. Effects of freezing and storage on the microestructure and textura of ewe's milk cheese. Zeitschrift für LebensmittelUntersuchung und -Forschung, 1996; 203(3): 245-251.

13. Gunasekaran, S., Ak, M.M. "Cheese Rheology and Texture". CRC Press LLC Boca Raton, Florida, 2003.

14. Spreer, E. "Lactología Industrial”, 2ª edición, Editorial Acribia. Zaragoza. España, 1991.

15. Torres, J. "Utilización del ultrafiltrado de suero pasteurizado del queso para el desarrollo de una bebida isotónica". Proyecto Especial del Programa de Ingeniero Agrónomo, Zamorano, Honduras. 2001.

16. Guerrero-Rodríguez W. J., Gómez-Aldapa C.A., Castro-Rosa J., González-Ramírez C.A., Santos-López E.M. "Caracterización Fisicoquímica del Lactosuero en el Valle de Tulancingo". XII Congreso Nacional de Ciencia y Tecnología de Alimentos. División Ciencias de la Vida. Campus Irapuato-Salamanca. México, 2010.

17. Instituto Nacional de Salud. "Tablas peruanas de composición de alimentos". Centro Nacional de Alimentación y Nutrición. Lima, Perú, 2009.

18. Demonte, P. "Evaluación sensorial de la textura y búsqueda de correlaciones con medidas instrumentales". Seminario de Textura y Reología de los Alimentos. Memorias. Cali: Universidad del Valle, 1995, 8-20.

19. Jaros D., Petrag J., Rohm H., Ulberth F. Milk fat composition affects mechanical and rheological properties of processed cheese. Applied Rheology, 2001; 11(1): 19-25.

20. Lewis, J. "Propiedades físicas de los alimentos y de los sistemas de procesado". Editorial ACRIBIA, S. A. Zaragoza. España, 1993.

21. Madureira A, Pintado I, Gomes A, Pintado E, Malcata, F. Rheological, textural and microstructural features of probiotic whey cheeses. LWT - Food Science and Technology, 2011; 44 (1): 75-81. 